\title{
Development of 1.6 GPa pressure-measuring multipliers
}

\author{
W. Sabuga ${ }^{1}$, R. Haines ${ }^{2}$ \\ ${ }_{1}^{1}$ Physikalisch-Technische Bundesanstalt, Bundesallee 100, 38116 Braunschweig, Germany \\ ${ }^{2}$ Fluke Calibration, 4765 East Beautiful Lane, Phoenix, AZ 85044-5318, USA
}

\section{ABSTRACT}

Two 1.6 GPa pressure-measuring multipliers were developed and built. Feasibility analysis of their operation up to $1.6 \mathrm{GPa}$, parameter optimisation and prediction of their behaviour were performed using Finite Element Analysis (FEA). Their performance and metrological properties were determined experimentally at pressures up to $500 \mathrm{MPa}$. The experimental and theoretical results are in reasonable agreement. With the results obtained so far, the relative standard uncertainty of the pressure measurement up to $1.6 \mathrm{GPa}$ is expected to be not greater than $2 \cdot 10^{-4}$. With this new development the range of the pressure calibration service in Europe can be extended up to $1.5 \mathrm{GPa}$.

\section{Section: RESEARCH PAPER}

Keywords: High pressure standards, pressure multipliers, finite element analysis, pressure transducers, calibration

Citation: Wladimir Sabuga, Rob Haines, Development of 1.6 GPa pressure-measuring multipliers, Acta IMEKO, vol. 3, no. 2, article 13, June 2014, identifier: IMEKO-ACTA-03 (2014)-02-13

Editor: Paolo Carbone, University of Perugia

Received April $15^{\text {th }}, 2013$; In final form August $16^{\text {th }}, 2013$; Published June 2014

Copyright: (C) 2014 IMEKO. This is an open-access article distributed under the terms of the Creative Commons Attribution 3.0 License, which permits unrestricted use, distribution, and reproduction in any medium, provided the original author and source are credited

Funding: This research was jointly funded by the EMRP participating countries within EURAMET and the European Union.

Corresponding author: Wladimir Sabuga, e-mail: wladimir.sabuga@ptb.de

\section{INTRODUCTION}

New high pressure technologies such as autofrettage, hydroforming and isostatic pressing are being intensively developed and used in the automotive industry, diesel engineering, vessel production for the petrochemical and pharmaceutical industry, water cutting machine manufacture, new material fabrication and, recently, for food sterilisation. New transducers for measuring pressures up to $1.5 \mathrm{GPa}$ have recently been developed and are offered by several manufacturers. The use of these high pressure transducers requires their calibration and, thus, existence of appropriate reference pressure standards traceable to the International System of units. The operation range of the pressure standards in West Europe is limited by $1.4 \mathrm{GPa}$. Creation of new primary pressure standards up to $1.6 \mathrm{GPa}$ and establishing a calibration service up to $1.5 \mathrm{GPa}$ is the objective of a Joint Research Project (JRP) "High pressure metrology for industrial applications" within the European Metrology Research Programme (EMRP) [1, 2]. PTB and Fluke Calibration (Fluke) have jointly developed and built two 1.6 GPa pressure measuring multipliers to extend the pressure scale and the calibration range as required.

\section{PRINCIPLE AND KEY FEATURES OF THE PRESSURE MULTIPLIERS}

The operation principle of a pressure measuring multiplier is explained in figure 1. The multiplier includes a low pressure $(\mathrm{LP}), p_{\mathrm{L}}$, and a high pressure (HP), $p_{\mathrm{H}}$, piston-cylinder assembly (PCA) which have significantly different effective areas. The LP and HP PCAs are axially aligned and their pistons are mechanically coupled. Both, LP and HP pistons are unsealed in the cylinders and are rotated, which, due the lubrication effect, avoids mechanical friction between the pistons and cylinders. Consequently, in the absence of other forces, the forces due to pressures $p_{\mathrm{H}}$ and $p_{\mathrm{L}}$ on the pistons are balanced when the ratio of pressures is equal to the ratio of the effective areas of the LP and HP PCAs, $A_{\mathrm{HP}}$ and $A_{\mathrm{LP}}$ :

$p_{\mathrm{H}} / p_{\mathrm{L}}=A_{\mathrm{HP}} / A_{\mathrm{LP}}$.

The high pressure, $p_{\mathrm{H}}$, can thus be determined by accurately measuring $p_{\mathrm{L}}$ and by knowing the exact ratio of $A_{\mathrm{HP}}$ to $A_{\mathrm{LP}}$, also called multiplying ratio $\left(K_{\mathrm{M}}\right)$. The principle of the pressure measuring multipliers has been in use at least since the 1930s and is utilised in current practice, for example in the $1.5 \mathrm{GPa}$ national pressure standard of Russia, VNIIFTRI [3]. A $1 \mathrm{GPa}$ pressure multiplier has been commercially offered since the late 


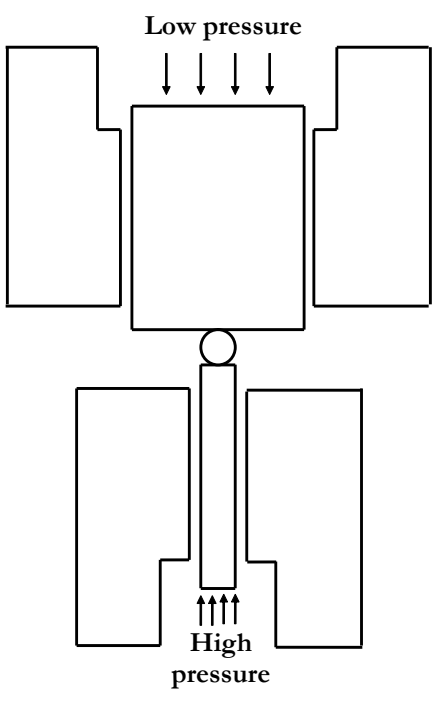

Figure 1. Operation principle of a pressure multiplier.

1980s and is used by national metrology institutes and calibration laboratories as a secondary or transfer standard [4].

In the newly developed multiplier, the nominal effective areas of the LP and HP PCAs were chosen to be $1 \mathrm{~cm}^{2}$ and $5 \mathrm{~mm}^{2}$, respectively. These are dimensions for which production technology is well established and that result in a pressure ratio of $1: 20$. Thus, a pressure, $p_{\mathrm{H}}$, of $1.6 \mathrm{GPa}$ on the $\mathrm{HP}$ side of the multiplier is reached at $p_{\mathrm{L}}=80 \mathrm{MPa}$ which is easily generated and measured with high accuracy. The design of the new multiplier has specific features which distinguish it from that of the former multipliers. First, to avoid plastic deformation and to guarantee stability of the effective areas the components of the LP and HP PCAs are made of tungsten carbide with $6 \%$ or $10 \%$ (HP piston) cobalt (WC-Co) instead of steel used in [3]. Since the tensile strength of the tungsten carbide is limited to roughly $0.7 \mathrm{GPa}$, the HP cylinder can be operated at the maximum pressure of $1.6 \mathrm{GPa}$ only if it is supported from outside. Thanks to a special design of the multipliers a compressive load is established on the outside of the HP tungsten carbide cylinder which prevents rupture when the pressure inside the cylinder exceeds the material tensile strength. In [4] this is accomplished by fitting a sleeve around the cylinder. In order to extend to $1.6 \mathrm{GPa}$ in the new multiplier, two sleeves, each made of chrome/nickel/molybdenum steel, are successively assembled onto the tungsten carbide HP cylinder by means of thermal shrink fits. In addition, the HP cylinder with the two sleeves is set into a jacket which allows a jacket pressure $\left(p_{j}\right)$ to be applied to the lateral surface of the outer sleeve and, thus, to additionally compensate the tensile stress in the cylinder (figure 2).

The HP PCA is designed to be operated in controlled clearance (CC) mode with $p_{j}$ typically equal to $25 \%$ of $p_{\mathrm{H}}$, at which the pressure distortion coefficient $(\lambda)$ of the PCA should be around zero. In addition, the HP PCA may be operated with variable $p_{\mathrm{j}}$ in order to adjust the piston fall rate $\left(\nu_{\mathrm{f}}\right)$ and the PCA sensitivity, if necessary, as well as to study $\lambda$ experimentally by measuring dependences of $v_{\mathrm{f}}$ and $A_{\mathrm{HP}}$ on $p_{\mathrm{j}}$. For optimal and stable operation of a PCA it is desirable that the pressure in the piston-cylinder gap changes linearly from its maximum value at the gap inlet to the ambient pressure at the gap outlet. Such a pressure distribution is difficult to realise in the case of CC HP
PCAs having a nominally constant gap in the pressure-free state because, under pressure, the piston-cylinder gap becomes extremely small in the outlet region due to a cross-sectional expansion of the axially loaded piston and a simultaneous reduction of the cylinder bore due to the jacket pressure [5]. In [3], where PCAs are operated in the re-entrant mode, which produces even stronger contraction of the cylinder than the CC mode, the problem was solved by giving the cylinder bore a flare-like shape with a diameter at the outlet being by few micrometers larger than at the inlet. Such a manufacture strategy is extremely difficult and generally leads to large widths and irregularities of the piston cylinder gap. In the new multiplier, the problem is overcome by giving the outer surface of the inner sleeve a variable shape. In the lower part, where the pressure inside the cylinder and in the PCA gap is much larger than the ambient pressure, the inner sleeve has a cylindrical shape. In the upper part, where the pressure in the gap approaches the ambient pressure, the inner sleeve has a conical shape with the diameter at the top being by $0.3 \mathrm{~mm}$ smaller than the diameter of the cylindrical part. This results in a tapered gap between the inner and outer sleeves which reduces the action of $p_{j}$ on the upper part of the inner sleeve. Therefore, excessive concentration of the pressure gradient in the pistoncylinder clearance towards the outlet of the cylinder is avoided and an acceptable flow rate of the pressure transmitting liquid between the piston and the cylinder is provided. The optimal shape of the inner sleeve was determined by Finite Element Analysis (FEA) as described in the next section.

The LP PCA was designed keeping in mind the requirement to have sufficiently low fluid flow through the piston-cylinder gap. This requirement results from the relatively large effective area of the LP PCA compared to the area of a pressure balance PCA maintaining and measuring $p_{\mathrm{L}}$. Usually, PCAs used in the range of $80 \mathrm{MPa}$ have nominal effective areas of $0.1 \mathrm{~cm}^{2}$, which is ten times smaller than $A_{\mathrm{LP}}$. Excessive flow rate in the LP PCA would cause a high piston fall rate of the reference pressure balance which could increase uncertainties or result in insufficient time for stable $p_{\mathrm{H}}$. To limit the flow rate and optimize performance, the principle of negative free

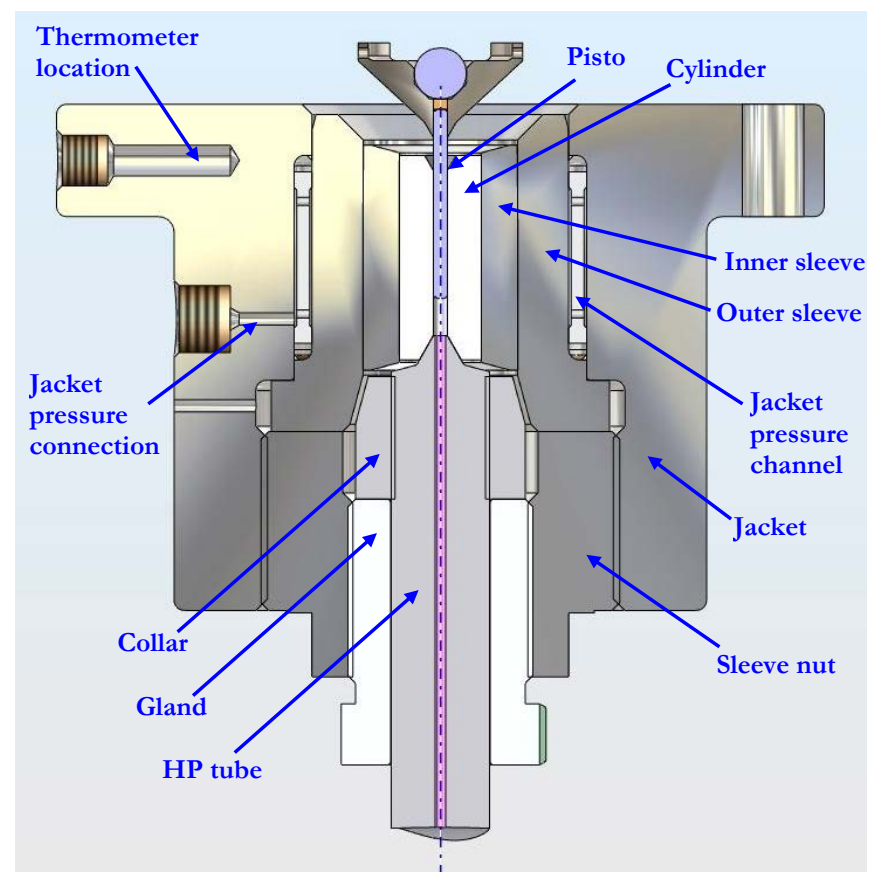

Figure 2. HP PCA in the mounting post. 
deformation was applied. This principle is well proven and is used in Fluke gas high pressure balances [6], providing low fall rates at higher pressures and high sensitivity at lower pressures. In the LP PCA design, the LP cylinder is surrounded by a sleeve with a conical taper on the inside surface and $p_{\mathrm{L}}$ is applied to the outside surface of the sleeve. The LP sleeve has a sliding fit on the cylinder and is positioned so that its smallest diameter is located where the cylinder pressure is maximal. In the absence of pressure, the sleeve produces no stress on the cylinder. As pressure increases, loading the cylinder from inside and the sleeve from outside, the sleeve first comes in contact with the cylinder in the region where the pressure in the pistoncylinder gap is maximal. In this way, a variable outside load of the cylinder is created that optimally compensates the radial distortion of the cylinder produced by the inner pressure. With this variable outside load distribution a nearly linear pressure distribution in the LP PCA gap is achieved.

\section{FINITE ELEMENT ANALYSIS}

To analyse feasibility of the pressure multipliers' operation up to $1.6 \mathrm{GPa}$ and to optimise dimensions of the PCAs components they were modelled using FEA. The modelling was performed using two different FEA software packages, ANSYS at PTB and Cosmos/Works at Fluke. In this way, correctness of the calculations was verified by analysing the same problem. Additionally, the analyses were performed for different problems to get complementary information on the multipliers performance. The FEA included large deflection, contact and plastic capabilities, the latter required for the tube connected to the HP PCA. First, all parts were modelled with their material properties and assumed geometries. For the HP PCA the shrinking of the inner and then of the outer sleeve on the tungsten carbide cylinder, and connection of the HP tube to the HP cylinder was modelled.

The cylinder to inner sleeve shrink fit was performed first, using nominal geometry. The deformation of the outer surface of the inner sleeve after this step was noted. In production this surface is re-machined after the initial shrink fit. To simulate this, the outer diameter of the inner sleeve was changed reducing it by the amount of deformation, to achieve geometry after this initial shrink step that gives a good representation of the geometry that results in production. The inner to outer sleeve shrink fit was performed second, using the resulting cylinder/inner sleeve combination with the outer sleeve nominal geometry. The shrink fit of the taper in the inner sleeve was accomplished in the same manner as the other shrink fit surfaces. The amount of contact of the surfaces was determined iteratively in the analysis, a step performed automatically by the FEA software. Three inner sleeve outside shapes were numerically tested in their effect on the stress, pressure distribution in the piston-cylinder gap and $\lambda$.

Connection of the HP tube to the cylinder and deformation of the tube under pressure were studied. A tube tip angle of $59.5^{\circ}$ and matching cylinder cone angle of $60^{\circ}$ were selected. The tube was moved into the cylinder to get a contact along the whole length of the cylinder cone and a pressure of 1.6 GPa was applied.

Surface loads in various combinations were applied. The loads included $50 \%$ or $100 \%$ of maximum measurement pressure on relevant surfaces, a linear and, alternatively, constant pressure distribution in the piston-cylinder gap, as well as a jacket pressure on the outer surface of the HP PCA sleeve
Table 1. Material properties.

\begin{tabular}{lcccc}
\hline Part / Material & $E / G P a$ & $\mu$ & $S_{\mathrm{y}} / \mathrm{GPa}$ & $S_{\text {ut }} / \mathrm{GPa}$ \\
\hline $\begin{array}{l}\text { LP PCA, HP } \\
\text { cylinder / }\end{array}$ & 620 & 0.218 & - & $\approx 0.7$ \\
\begin{tabular}{l} 
WC-6\%Co \\
\hline $\begin{array}{l}\text { HP piston / } \\
\text { WC-10\%Co }\end{array}$
\end{tabular} & 560 & 0.218 & - & - \\
\hline $\begin{array}{l}\text { LP \& HP sleeves / } \\
\text { Cr-Ni-Mo steel }\end{array}$ & 200 & 0.3 & 1.2 & 1.4 \\
\hline $\begin{array}{l}\text { HP tube / austenitic } \\
\text { steel }\end{array}$ & 200 & 0.3 & 1.053 & 1.216 \\
\hline
\end{tabular}

and a LP on the outer surface of the LP PCA sleeve. After each load step, radial deformation, radial and tangential stresses were extracted.

The stress and strain distributions were analysed in relation to the ultimate tensile strength $\left(S_{\mathrm{ut}}\right)$ and the elastic limit $\left(S_{\mathrm{y}}\right)$ of the cylinder, sleeve, and HP tube materials. These properties together with the Young's modulus $(E)$ and the Poisson ratio $(\mu)$ based on the information by the materials' manufacturers and literature data are compiled in table 1 . In addition, the ultimate compressive strength of the WC materials is known to be extremely high of about $7 \mathrm{GPa}$. Later, $E$ and $\mu$ values were accurately measured using resonant ultrasound spectroscopy [7].

After the shrink fit of the inner sleeve on the cylinder, a tangential stress of about $-600 \mathrm{MPa}$ (compression) was achieved at the inside of the cylinder. The tangential stress distribution at the cylinder inside after the subsequent shrinking fit of the outer sleeve is shown in figure 3. In any point, the absolute value of the stress is much lower than the ultimate compressive strength of the WC materials (7 GPa). In the upper part of the PCA, the absolute value of the stress becomes lower which results from the conical shape of the outer surface of the inner sleeve. This corresponds to the intended reduction of the outside support in the region of the internal pressure drop. The dashed line in figure 3 shows the tangential stress calculated analytically under assumption of cylindrically perfect cylinder and sleeves. Both the FEA and analytical results demonstrate that the double shrink will to a great extent compensate for the stress produced by the internal pressure of $1.6 \mathrm{GPa}$. For a linear pressure distribution in the gap the maximum residual stress produced by the shrinking and the internal pressure would be about $400 \mathrm{MPa}$, which could be withstood by the WC cylinder even in the absence of jacket pressure. However, in order to minimize risk of cylinder rupture, jacket pressure is expected to

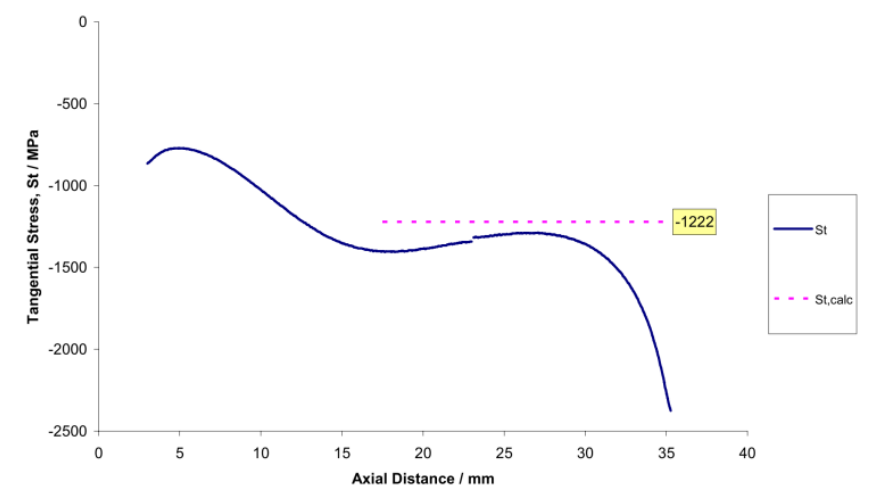

Figure 3. Tangential stress at the HP cylinder inside after shrinking fit of 2 sleeves calculated with FEA $\left(S_{t}\right)$ and analytically $\left(S_{t, \text { calc }}\right)$. 


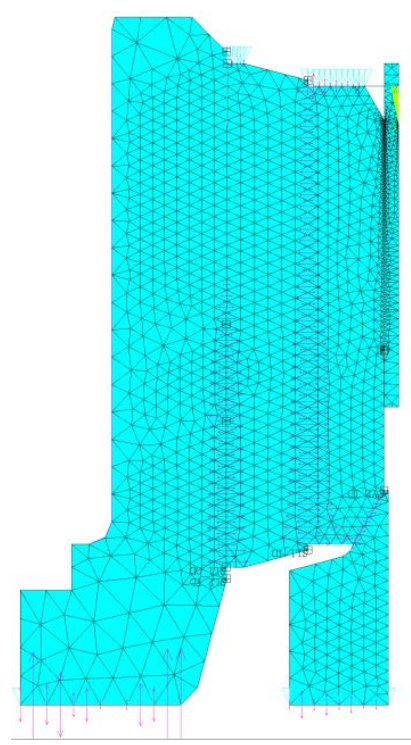

a

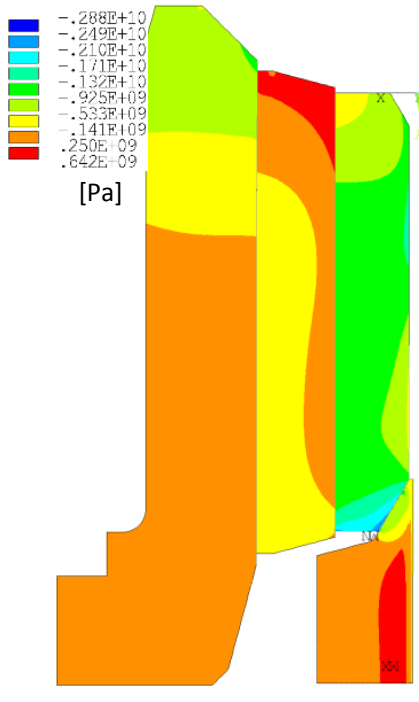

b
Figure 4. FEA model of HP PCA (a), tangential stress distribution in it at $p_{\mathrm{H}}=1.6 \mathrm{GPa} \& p_{\mathrm{j}}=0.4 \mathrm{GPa}(\mathrm{b})$.

be applied in all normal system operation.

Figure 4 presents the FEA model of the HP PCA and tangential stresses in the PCA components at $p_{\mathrm{H}}=1.6 \mathrm{GPa}$, a linear pressure distribution from 1.6 $\mathrm{GPa}$ to zero along the piston-cylinder gap and $p_{\mathrm{j}}=0.4 \mathrm{GPa}$. The FEA calculations for the HP PCAs at the maximum measurement pressure of 1.6 $\mathrm{GPa}$ and the jacket pressure of $400 \mathrm{MPa}$ show that the radial and tangential stress distributions are smooth and without any significant concentrations, the cylinder is subject only to compressive stresses, and the sleeves remain within the elastic limit. These results for the HP PCA indicate that the design is not at risk for cylinder rupture nor instability of the effective area due to plastic deformation in the sleeves. The calculations also confirm the necessity of having two sleeves on the HP cylinder in order to achieve the required cylinder compression when the temperature for the thermal shrink is kept below the tempering temperature of the sleeve material.

The analysis of the tube demonstrates that only a small portion of the tube near the center line is subject to plastic deformation. In the tapered part of the tube, in the sections where the tube is not supported by the cylinder, the region of plastic deformation does not exceed $1 / 3$ of the tube cross section. These results indicate a reliable connection between the HP PCA and tube at pressures up to $1.6 \mathrm{GPa}$. It is necessary to note that the FEA was performed assuming the ultimate strength and the elastic limit each to be the same for tensile and compressive deformations based on the available manufacture data. This might produce inaccuracies of the FEA results if these material properties were different for tension and compression.

In a similar manner as the HP PCA, an FEA of the LP PCA was performed at $p_{\mathrm{L}}=80 \mathrm{MPa}$ and a linear pressure distribution from $p_{\mathrm{L}}$ to zero being applied to inside of the cylinder with $p_{\mathrm{L}}$ applied to the outside of the sleeve surrounding the cylinder. The primary objective was to minimise the radial distortions at the cylinder inside and thus the fluid flow rate. It was found out that, with an optimal taper on the inside of the sleeve, the radial distortions of the cylinder do not exceed $0.1 \mu \mathrm{m}$ at any point of the cylinder bore. Without the sleeve and in the free deformation (FD) mode, they would reach $1 \mu \mathrm{m}$ at the gap entrance.

Combining the structural FEA of the HP PCA with a hydrodynamic analysis for its piston-cylinder gap $\lambda$ and $\nu_{\mathrm{f}}$ were calculated with using the PTB iterative method described in [5]. As a pressure transmitting medium two liquids were considered: di(2)-ethyl-hexyl-sebacate (DHS) at $p_{\mathrm{H}} \leq 0.5 \mathrm{GPa}$ and polydiethylsiloxan PES-1 for $p_{\mathrm{H}} \leq 1.6 \mathrm{GPa}$. DHS is a liquid widely used in pressure balances up to $1 \mathrm{GPa}$. However DHS is not applicable at higher pressures because of solidification. Its density and viscosity dependences on pressure were used as given e.g. in [5]. PES-1 has a significantly lower viscosity than DHS with acceptable values up to $1.6 \mathrm{GPa}$. Its density and viscosity as functions of pressure were based on the experimental data presented in [3]. With DHS, calculations were performed in FD mode to provide target values of $v_{\mathrm{f}}$ for optimal piston-cylinder gap widths to be achieved in the pistoncylinder production process. With PES-1, both FD and CC modes were analysed. As known from former FEA studies results of hydrodynamic modelling strongly depend on a real initial gap profile between undistorted piston and cylinder [5]. In particular, information about the cylinder bore profile near the exit is important because the gap in this region becomes the narrowest under high pressure and therefore has a strong effect on the pressure distribution, $\nu_{\mathrm{f}}$ and $\lambda$. To take this into account, prior to performing a final adjustment of the piston to the cylinder bore in the multiplier production process described in section 4 , dimensional measurements were performed on the two HP cylinders. They included straightness measurements in the outlet region of the cylinder bore along 4 generatrix lines separated by $45^{\circ}$. Results for opposite generatrices $\left(0^{\circ}\right.$ and $180^{\circ}, 45^{\circ}$ and $225^{\circ}$, and so on) were averaged and are shown for the two cylinders in figure 5 .

For FEA calculations, where the PCAs are treated as axisymmetric, the gap profiles were averaged and approximated by analytical functions which are also presented in figure 5 . The piston and the cylinder bore apart from the gap exit were considered ideally cylindrical. Different gap widths (b) were analysed. Figure 5 presents the case in which $b$ was equal to $0.2 \mu \mathrm{m}$.

Results of the piston fall rate calculations for $h=(0.2-0.5) \mu \mathrm{m}, \mathrm{FD}$ and CC operation modes, DHS and PES1 liquids are shown in figure 6.

Even with the smallest technologically feasible gap of $0.2 \mu \mathrm{m}$ $v_{\mathrm{f}}$ is too high when PES-1 and FD mode are used. The largest gap considered, $0.5 \mu \mathrm{m}$, combined with CC mode produces

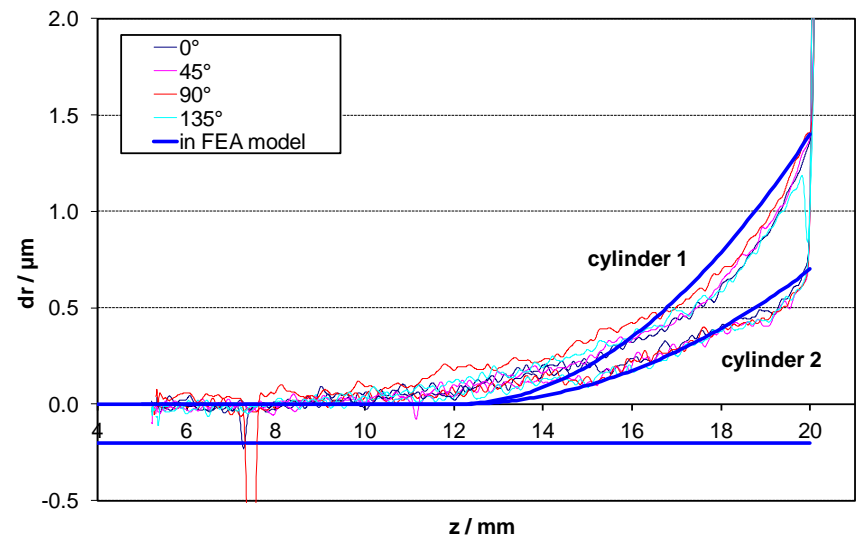

Figure 5. Piston-cylinder gap near the outlet for a perfect piston and real dimensions of cylinders 1 and 2 . 


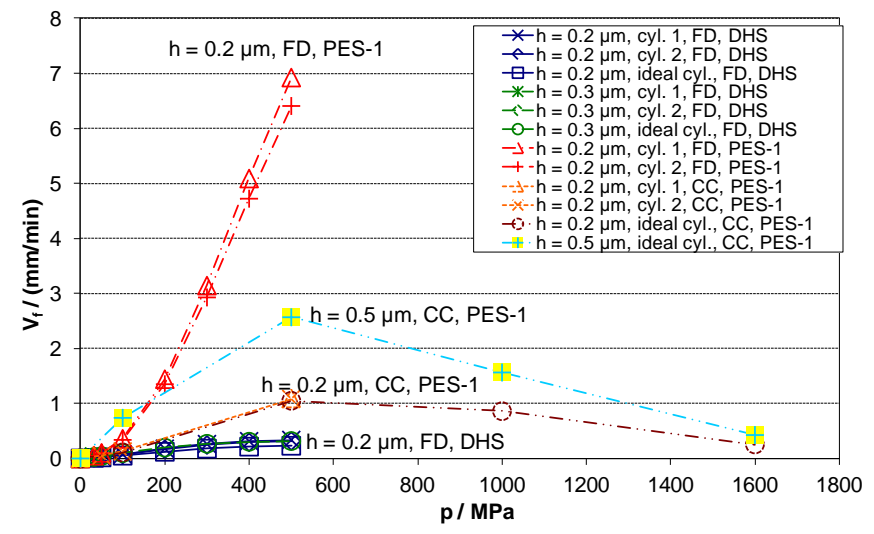

Figure 6. Piston fall rates calculated for different gap sizes, profiles and liquids.

acceptable $v_{\mathrm{f}}$ at $p_{\mathrm{H}}>1 \mathrm{GPa}$ but rates that are still too high below $1 \mathrm{GPa}$. Surprisingly, the difference between the piston fall rates for $0.2 \mu \mathrm{m}$ and $0.5 \mu \mathrm{m}$ gaps in the pressure range (1 to 1.6) GPa is not as big as would be expected from theory of the undistorted gap. With the FEA results for $v_{\mathrm{f}}$ the range $b=(0.3-$ $0.4) \mu \mathrm{m}$ was found as optimal. With $h=0.4 \mu \mathrm{m}$, a target $\nu_{\mathrm{f}}$ of (0.068 to 0.073$) \mathrm{mm} / \mathrm{min}$ was defined to be achieved at $32 \mathrm{MPa}$ in control measurements when fitting the pistons to the cylinders. At $500 \mathrm{MPa}$ with DHS and in FD mode, this gap width leads to $v_{\mathrm{f}}=(0.57-0.61) \mathrm{mm} / \mathrm{min}$.

\section{REALISATION OF THE MULTIPLIERS}

For both the HP and LP PCAs, the best designs indicated by the FEA were realized. Complementary technologies available at PTB and Fluke were combined. The piston-cylinders were manufactured and detailed technical drawings of the multipliers and all their parts produced by Fluke. All other parts - each of the two multipliers comprised about 80 parts - were manufactured by PTB. Fluke carried out a final mechanical adjustment of the sleeves and some other parts. In particular, processing of the final diameters of the sleeves to meet the defined tolerances of $1 \mu \mathrm{m}$ and to achieve a roughness of lateral surfaces better than $0.2 \mu \mathrm{m}$ required Fluke's expertise.

The production of the PCAs started with 5 to 8 pieces of LP and HP pistons and cylinders as well as sleeves. The best were selected during the succeeding processing and characterisation.

PTB performed dimensional measurements and performed the thermal shrink fits of the sleeves on the cylinders. For the shrink fits, the outer steel sleeve was heated up to $400{ }^{\circ} \mathrm{C}$ maximum to stay below the tempering temperature of the sleeve's steel, which is $450{ }^{\circ} \mathrm{C}$. However, provisional shrinking trials indicated that $400{ }^{\circ} \mathrm{C}$ of temperature increase may not be sufficient to perform the shrink - the insert stuck in the outside sleeve. To get more space and time leeway for the shrinking procedure, a greater temperature difference between the two parts was created by cooling the insert (cylinder in the $1^{\text {st }}$ shrink stage and cylinder with already shrunk inner sleeve in the $2^{\text {nd }}$ shrink stage) down to about $-196{ }^{\circ} \mathrm{C}$ using liquid nitrogen. Prior to shrinking the outer sleeve onto the inner, which had been fit to the cylinder in the $1^{\text {st }}$ shrink, the cylindrical and conical outside surfaces of the inner sleeve was characterised dimensionally.

After the two HP cylinders heat shrink operations their bores were re-machined to remove 3 to $5 \mu \mathrm{m}$ from inner surfaces deformed by shrinking. The HP pistons and cylinders were then lapped to achieve piston fall rates which had been predicted by FEA with the gap width of $h=(0.3-0.4) \mu \mathrm{m}$. The test piston fall rate measurements in the production stage were performed at a pressure of $32 \mathrm{MPa}$ at which the effect of the elastic distortion is relatively small and $v_{\mathrm{f}}$ primarily depends on the undistorted gap width. Later, piston fall rates were measured in both HP and LP PCAs and allowed estimation of the gap width between piston and cylinders. It was found $h=(0.27-0.36) \mu \mathrm{m}$ for the HP PCAs and $h=(0.68-0.73) \mu \mathrm{m}$ for the LP PCAs.

The whole production required the multipliers' parts to be sent between PTB and Fluke, some of them many times, for the subsequent production, characterisation and adjustment procedures. Finally, the multipliers were assembled and preliminarily tested by Fluke.

\section{EXPERIMENTS}

First tests of the multipliers were performed by Fluke at pressures (100 to 500) $\mathrm{MPa}$ on the HP side and (5 to 25) $\mathrm{MPa}$ on the LP side of the multipliers using two piston gauges as a reference, with DHS as a pressure transmitting liquid and at $p_{\mathrm{j}}=0.25 \cdot p_{\mathrm{H}}$. The setup is shown in Figure 7.

Multiplying ratios were determined using two hydraulic pressure balances in a crossfloat. Both LP and HP piston gauges were PG7302. Two different $500 \mathrm{kPa} / \mathrm{kg}$ PCAs having expanded uncertainties in pressure of $22 \cdot 10^{-6} \cdot p_{\mathrm{L}}+16 \mathrm{~Pa}$ and $27 \cdot 10^{-6} \cdot p_{\mathrm{L}}+16 \mathrm{~Pa}(k=2)$ were used in different runs on the LP side. A $5 \mathrm{MPa} / \mathrm{kg}$ PCA having expanded uncertainty in pressure of $70 \cdot 10^{-6} \cdot p_{\mathrm{H}}+16 \mathrm{~Pa}(k=2)$ was used on the HP side of the multiplier. The HP and LP PCAs' temperatures in the multiplier were measured using platinum resistance thermometers. These temperatures and the pistons position in the multiplier were indicated by a laboratory conditions monitor (LCM). The pistons were kept within $\pm 2.5 \mathrm{~mm}$ around their middle working position. They were rotated by a DC motor at approximately $10 \mathrm{rpm}$. A PPCH hydraulic pressure controller was used to set $p_{\mathrm{j}}$. A tare pressure $\left(p_{\mathrm{T}}\right)$ produced on the HP side of the multiplier by the masses loading the HP piston (HP and LP pistons, pistons coupler, etc.) was measured at $p_{\mathrm{L}}=0$ for each multiplier assembly using an RPM3 A1000, H1 (0-2) MPa pressure monitor with an uncertainty of approximately $2 \mathrm{kPa}$ $(k=2)$. It was equal to $(2.918$ and 2.926$) \mathrm{MPa}$ for the two multipliers. With the tare pressure equation (1) transforms to $p_{\mathrm{H}}=p_{\mathrm{T}}+p_{\mathrm{L}} \cdot K_{\mathrm{M}}$, with

$K_{\mathrm{M}}=K_{\mathrm{M}, 0} \times\left[1+\lambda_{K^{\mathrm{M}}} \cdot\left(p_{\mathrm{H}}-p_{\mathrm{T}}\right)\right]$,

where $K_{\mathrm{M}, 0}$ is $K_{\mathrm{M}}$ at $p_{\mathrm{L}}=0$ and $\lambda_{\mathrm{W}}$ is the pressure dependence coefficient of $K_{\mathrm{M}}$. A crossfloat, using the drop rate method, was performed between the two piston gauges with multiplier either 1 or 2 in between the two piston gauges at $p_{\mathrm{H}}=(100,200,300$,

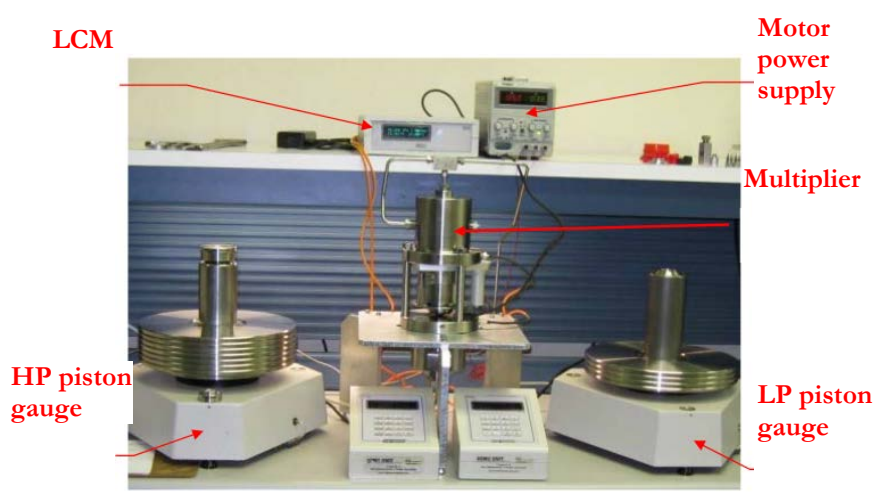

Figure 7. Multiplier system test setup. 


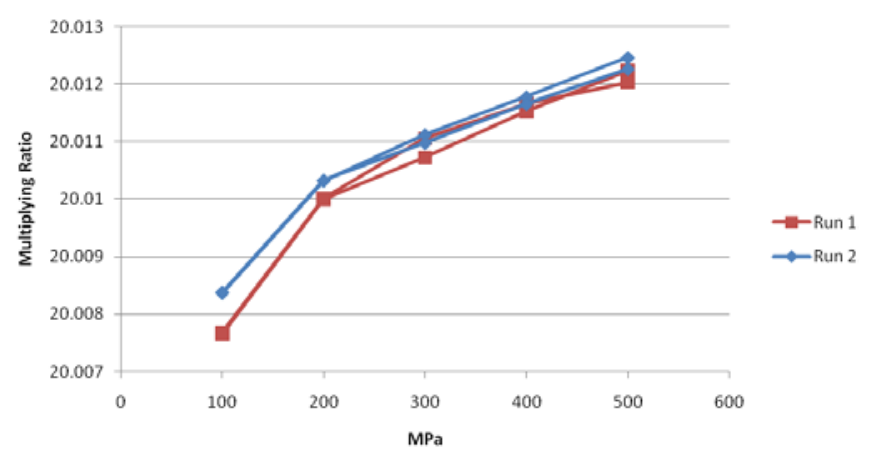

Figure 8. Multiplying ratio vs. high pressure corrected for tare pressure.

$400,500,500,400,300,200,100) \mathrm{MPa}$ in four runs total. According to (2), the multiplying ratio was determined at each point by subtracting $p_{\mathrm{T}}$ from $p_{\mathrm{H}}$ measured on the HP side and dividing by $p_{\mathrm{L}}$ measured with the LP piston gauge (figure 8).

The set of data for each run was fit with function (3) providing $K_{\mathrm{M}, 0}$ and $\lambda_{K \mathrm{M}}$. The results of the two runs for each multiplier were combined (averaged) and used to determine the residuals of the points taken. After reviewing the results of the tests it was decided to leave out the $100 \mathrm{MPa}$ in determining $K_{\mathrm{M}, 0}$ and $\lambda_{K \mathrm{M}}$ as it did not seem typical with respect to the rest of the results. Table 2 gives the results of the fit for each multiplier.

Performance of the multipliers has been found satisfactory. The $K_{\mathrm{M}}$ values were reproducible within $\pm 4 \cdot 10^{-5}$ for multiplier 1 and $\pm 2 \cdot 10^{-5}$ for multiplier 2 . An increased standard deviation in the case of multiplier 1 is presumably associated with the exchange of the reference LP piston gauge between runs 1 and 2. Herewith and taking into account the uncertainties of the reference LP and HP piston gauges PG7302, the relative standard uncertainty of the multipliers in the pressure range $(100$ to $500 \mathrm{MPa}$ ) lies between (4 and 5.5$) \cdot 10^{-5}$. With the same data the relative standard uncertainty in the range (1 to 1.6) GPa can be expected to be $(1.3$ to 2$) \cdot 10^{-4}$, which is a very preliminary estimation and must be confirmed by experiments at higher pressures. This uncertainty is sufficiently small to provide a calibration service required by the industry.

\section{CONCLUSIONS AND OUTLOOK}

The two novel 1.6 GPa pressure-measuring multipliers were developed, tested at pressures up to $500 \mathrm{MPa}$, and demonstrated repeatability on the level of as low as $2 \cdot 10^{-5}$. A standard uncertainty of up to $5.5 \cdot 10^{-5}$ obtained in the test crossfloats is mainly caused by the reference LP and HP standards. This uncertainty can be reduced in the future by more extensive experiments using more accurate $1 \mathrm{GPa}$ standards of PTB as a reference, but also by a theoretical calculation of the pressure distortion coefficients of the LP and HP PCAs taking into account the real dimensional properties of
Table 2. Results of multiplying ratios in individual tests and averages for each multiplier.

\begin{tabular}{lcccc}
\hline & \multicolumn{2}{c}{ Multiplier 1 } & \multicolumn{2}{c}{ Multiplier 2 } \\
\cline { 2 - 5 } & $K_{\mathrm{M}, 0}$ & $\lambda_{K \mathrm{M}} \cdot 10^{7} \mathrm{MPa}^{-1}$ & $K_{\mathrm{M}, 0}$ & $\lambda_{K \mathrm{M}} \cdot 10^{7} \mathrm{MPa}^{-1}$ \\
\hline Run 1 & 19.987668 & 3.79 & 20.008672 & 3.55 \\
Run 2 & 19.989101 & 2.89 & 20.008994 & 3.39 \\
Average & 19.988384 & 3.34 & 20.008833 & 3.47 \\
\hline
\end{tabular}

the HP piston-cylinder gap and of sleeve-to-cylinder gap in the LP PCA. Moreover, extension of the fluid flow calculations for the PCA gap up to 1.6 GPa requires accurate data on density and viscosity of PES-1 at high pressure. All these measurements are in progress within EMRP JRP [1].

\section{ACKNOWLEDGEMENT}

The contribution of Dr. P. Ulbig in the organisation of this research and of Mrs. D. Hentschel, who manufactured most of the parts of the multipliers, both PTB members, is much appreciated. The authors acknowledge Mr. P. Delajoud (Fluke DHI retired) for the design of the multipliers and F. Valenzuela and M. Bair (both Fluke) for piston-cylinder fabrication and cross-float testing/analysis respectively. This research was carried out within the EMRP. It is jointly funded by the EMRP participating countries within EURAMET and the European Union.

\section{REFERENCES}

[1] EURAMET, "High pressure metrology for industrial applications", Publishable JRP summary report for IND03 HighPRES,

http://www.euramet.org/index.php?id=emrp_call_2010.

[2] EMRP JRP IND03 HighPRES, http://emrp-highpres.cmi.cz/.

[3] V.M. Borovkov, "Deadweight high pressure piston manometers", in: Researches in the Area of High Pressures. E.V. Zolotyh (editor). Publisher Izdatelstvo Standartov, Moscow, 1987, pp.577 , russ.

[4] P. Delajoud, "The pressure multiplier: a transfer standard in the range 100 to $1000 \mathrm{MPa}$ ", BIPM Monographie, vol. 89/1, 1989, pp.114-124.

[5] W. Sabuga et al., "Finite element method used for calculation of the distortion coefficient and associated uncertainty of a PTB 1 GPa pressure balance - EUROMET project 463", Metrologia, 43 (2006) pp.311-325.

[6] P. Delajoud, M. Girard, "A new piston gauge to improve the definition of high gas pressure and to facilitate the gas to oil transition in a pressure calibration chain", Proc. of the IMEKO TC16 Int. Symp. on Pressure and Vacuum, Sept. 22-24, 2003, Beijing, China, Acta Metrologica Sinica Press, pp.154-159.

[7] W. Sabuga, P. Ulbig, A.D. Salama, "Elastic constants of pressure balances' piston-cylinder assemblies measured with the resonant ultrasound spectroscopy", Proc. of the Int. Metrology Conf. CAFMET-2010, April 2010, Cairo. 